\title{
Study on an Available System by the Portable GPS for the Formation of Resident Participation to a Refuge Planning Scenario
}

\author{
Tohru FutagamiI ${ }^{1}$, Noboru Kimata ${ }^{2}$
}

\begin{abstract}
We have developed a visual simulation system based on construction of scenario Petri Net with regional map in the background to support resident participation for refuge planning. In this study, we propose to use portable GPS and construct basic part of refuse scenario Petri-Net directly based on GPS data of residents behavior in order to support more direct participation. For this task, we develop a procedure system to generate a file called as Petri Ndata based on GPS data of residents behavior and to convert the file into the Sdata file and demonstrate this procedure system for refuse planning in an intermediate mountainous area.
\end{abstract}

Keywords : Resident participation support, Refuge planning, Scenario simulation, Petri Net, Portable GPS measurement data

\section{Introduction}

According to Central Disaster Prevention Council ${ }^{12)}$ "Outline of Comprehensive Emergency Drill" in 2006, the purpose of the emergency drill is determined as the functional check of disaster prevention organization control, verification of effectiveness, the upsurge of national awareness of disaster prevention and improvement in knowledge, and study and education of a disaster prevention person in charge. Furthermore, promotion of training practical and effective as a basic policy which is in charge of emergency drill implementation, evaluation of training, positive training support of the country, etc. mention the device of the emergency drill which contributes to the national movement which reduces disaster damage. That is, the necessity for training which leads to decrease disaster which is the purpose of an emergency drill is described clearly. For that purpose, it will be the requisite that the residents who are a basis of support of public support and the leading role as it is in fundamental principles clarify the division of roles of collaboration, such as self-help and mutual aid.

Next, disaster is imagined concretely and the device of an emergency drill which can act exactly is needed. Some refuge simulators ${ }^{3)-6)}$ are developed as these tools.
However, they are not aiming at the support of the emergency drill such as residents leading role, because they only give visibility to output. On the other hand, we have been developing the scenario simulator ${ }^{7)-10)}$ aiming at support of public involvement. Concretely, we compose a simulator using a Petri Net, because a description principle is simple and clear. Therefore, it is easier for residents to understand the proposed plan. This can be said to be the system equipped with the ease of extension and correction of the plan by the basis of the visibility on a background image.

If residents participation is discussed on three levels, under the present circumstances, our system will be proved as a level 1 that an opinion and proposal of residents can fully be pulled out. As a level 2, it is proven also about feedbacks of resident's opinion and suggestion to the plan in no time, such as reflection and examination. In this study, we would like to give direct inclusion for the scenario simulation of residents' refuge act as public involvement of a level 3.

Therefore, the purpose of this study is to expand our system to more practical level such as direct resident participation by the developing technique of the evacuation route scenario Petri Net with portable GPS.

1) Member of JSCE, Ph.D., Lecturer, Center for Information Technology, Ehime University (3,Matsuyama Bunkyou, Ehime 790-8577, JAPAN)

2) Member of JSCE, D.E., Professor Emeritus, Kanazawa University (920-1192, Kakumatyou, Kanazawa) 


\section{Outline of Petri Net Simulation of Disaster Prevention Planning Scenario on Background Image}

\section{(1) The basic principle of a Petri Net}

The basic concept composition of a refuge scenario is shown in Fig.1-1.The residents who are present in a residence start refuge in response to refuge information (preparation, advice, and directions), and this figure expresses the situation which moves to the evacuation area of the destination through an evacuation route. The arrow from under a figure to the upper route is prevention disaster, and expresses prevention of such a landslide or a road broken by slope collapse.Fig.1-2 shows the example which described the basic concept composition of the refuge scenario of Fig.1-1 by the Petri Net. In a Petri Net, it is the Place group which is shown by $\bigcirc$ or $\square$ all over the figure, and | is a Transition group, with an arc, the former shows input-and-output relations and, the latter shows deterrence relations. Next, the token is shown by on Place and it directs the present state of an object system. The ignition rule of the transition shown below bears the drive principle of this token.

1) When marking of the token is carried out to input Place and a token exists in all the deterrence Place, the transition concerned firing.

2) One token is erased at a time from the input Place group, and one token is arranged at each output Place group.

Place P0 (it is described as the following P0) expresses the residence with the example of description of the refuge scenario by the Petri Net of Fig.1-2, and the token struck by $\mathrm{P} 1$ expresses the information on a refuge start with it. The token struck by P0 expresses the number of the residents who take refuge, and one resident has become a candidate for refuge in this figure P0.The present condition in Fig.1-2, since the token is struck by P0, P1, T0 become firing by the firing rule mentioned above, and a token moves to P2. If place timer PT2 and PT3 which is given to $\mathrm{P} 2$ and $\mathrm{P} 3$ respectively are consumed, firing of transition will take place. Corresponding with the basic concept composition of the refuge scenario of Fig.1-1, PT2 and PT3 are equivalent to the travel time between T0 and $\mathrm{T} 1$ and between $\mathrm{T} 1$ and $\mathrm{T} 2$, respectively.

Fig.2 has added proactive / reactive measure network to the example of description of the refuge scenario by the Petri Net of Fig.1-2 with the addition of disaster prevention. The portion surrounded with the dashed line is a basic network of Fig.1-2.

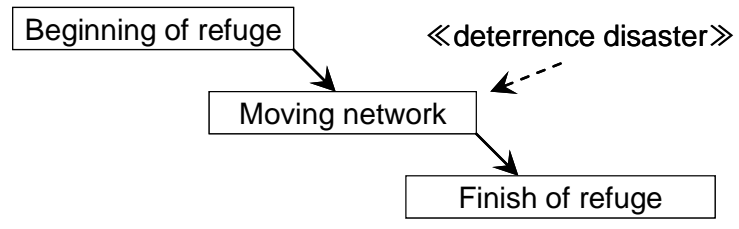

Fig.1-1 Basic Concept Composition of Refuge Scenario

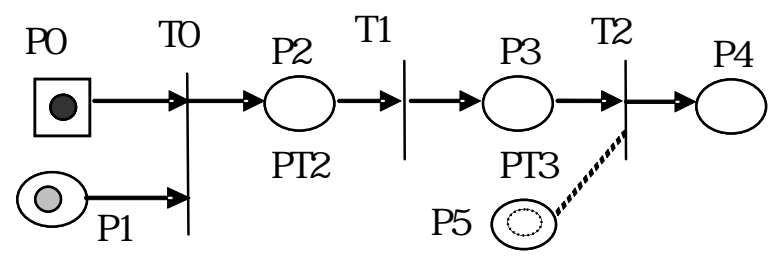

Fig.1-2 Example of Description of Refuge Scenario by Petri Net

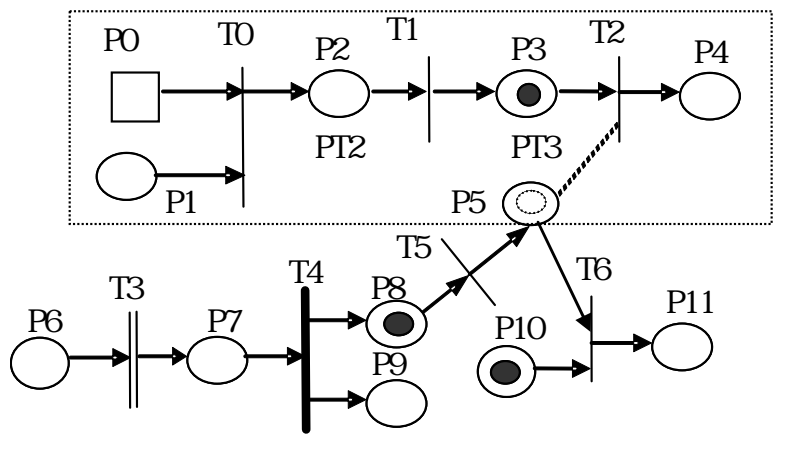

Fig.2 Addition of Disaster Prevention of Proactive / Reactive Measure

When the deterrence arc is applied to T2 and token is struck into P5 from a firing rule by Fig.1-2, deterrence starts T2 and it does not become movement to P4, i.e., the end of refuge. Then, T3 which considers P6 as the occurrence of an earthquake and is described by a double line expresses color generation selection transition, and a color token generates it probable in P7. The rate of incidence of this color token can be given exogenously.

Next, T4 expresses selection transition and a token is chosen as P8 or P9 by the color token attribute. If a token is struck by P8, a token will move to P5 and deterrence will start T2.That is, it means that prevention damage had occurred. On the contrary, if a token is struck by P9, deterrence of $\mathrm{T} 2$ will not occur but will mean a slight disaster. The portions which consist of T6, P10, and P11 will be prevention countermeasures against calamities, and if the token is arranged in advance to P10, even if a token moves to P5, T6 ignites, and a prevention token will disappear and will be outputted to P11. That is, after it 
means a prior measure and after a token moving to P5, the posteriori measure which arranges the token of P10 can be carried out by making network easily. As mentioned above, description of refuge and the prevention by a Petri Net is simple, so we can understand that it is clear and the drive principle is also easy. Furthermore, we can also understand easily the additional nature and the extendibility of the addition of prevention, and net description corresponding to disaster prevention planning called a prior / posteriori measure. As mentioned above, it turns out that the deployment nature and understanding nature called the addition of disaster information systems, such as generation and selection, have the feature on the basis of the common element, common structure, and common drive principle which is a drive principle of a Petri Net.

\section{(2) The outline of a Petri Net simulator}

In order to perform the Petri Net simulator which we have developed, it is necessary first to create Sdata. Sdata is the data file which drew the net figure modeled using Petri Net theory with the form adapted to a Petri Net simulator. The structure is the number of Place, the number of transition, each deterrence situation, a setup of each place, an input-and-output relation of the Place transition, generating Place's setup, etc. When the simulation is performed, it has described required data according to text format. The Sdata description by the Petri Net of Fig.1-2 is shown in Fig.3. The portion shown in the gray in Figure.3 prepares the line for each total under PLACE and TRAN in the basic format of Sdata, and specification description of the transition is carried out with Place. Partial (1) surrounded with the top dashed line in Fig. $\mathbf{3}$ is Place's description part. The description portion of each Place characteristic is outlined. The 2nd line describes a deterrent description in the 1st row. From candidate Place, if there is no deterrence, it will be described as -1 in the 2 nd row which shows the end of a deterrent description. From the 2nd line to the 5th line does not have deterrence, it becomes blank. The value of place timer is set as the 3rd row. The kind of color of a token is described to the 4th row.

When continuing from Place who existed before, it is described as 0 . The 5 th row is setup of a display and not displaying of a token, when displaying, it describes it as 1 , and it describes it as 0 at the time of un-displaying. The Place form will become $\mathrm{O}$ if it is described as 1 . It is referred to as 2 in the 2nd line, and since it is specified as 99 here, the Place form becomes $\square$. The 4 or 5 th line is course Place's setup about refuge information, and the 3rd line has set place timer as the 3rd row. Place timer is the

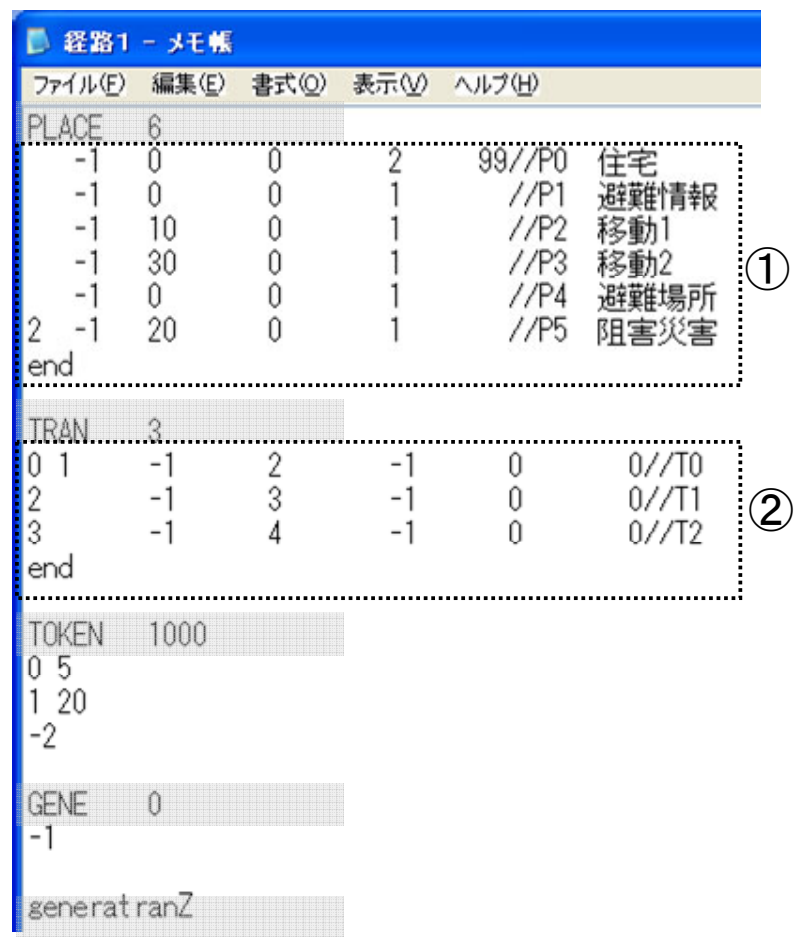

Fig.3 Sdata Formal Description of Petri Net

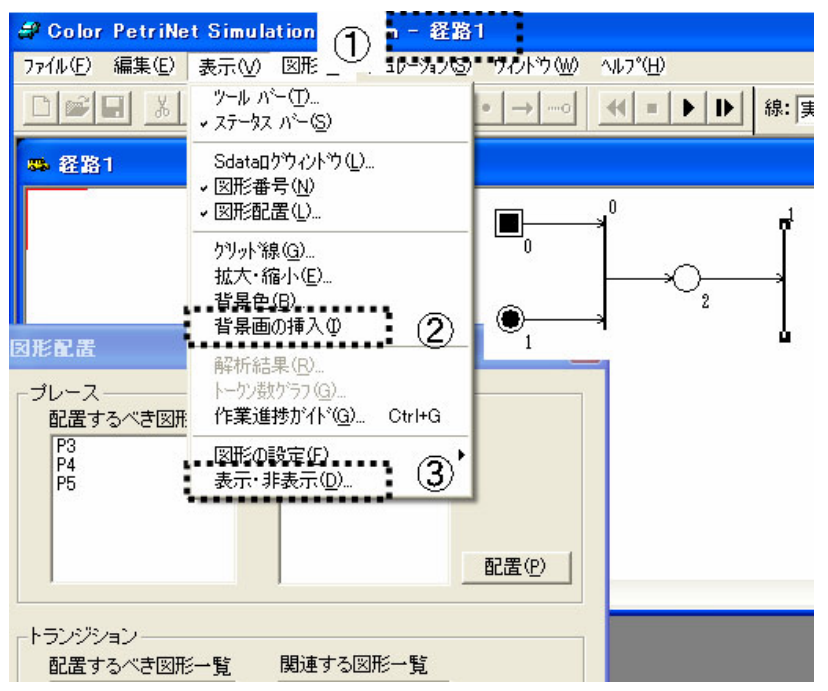

Fig.4 Basic Menu of Petri Net Simulator

travel time between the transition before and behind Place, and it sets up by breaking the numerical difference of a distance difference by average travel time. First Fig. 2 of the 7th line means that a deterrence arc is applied to T2 (transition number 2) from P5. The last "//" of each subsequent line describes a comment. “end" expresses declaration of the end of description of each place characteristic.

Next, lower dashed line part (2) of Fig. 3 is description 
about transition. It is in random order in the Place number inputted from object transition, and a space is inserted, and it describes to continuation, and, finally is described as -1 .

The 3rd row describes an output Place number from object transition, and, finally describes it as -1.If object transition is ordinary transition, the 5th row is 0 and color generation transition and it is 1 and color selection transition, it will describe it as 2.The 6th row is setup of a display and not displaying of transition, and is set to 1 in a display, and 0 in not displaying. In the following, the order of the real way which used the simulator is explained. Firstly, Course1.Sdata which uses a memo pad / Excel sheet is shown in Fig.3 is described. At this time, extension ".Sdata" is attached to a file name and it saves by text format. Next, simulator executable file "Petri.exe" is started and course1.Sdata is read. If it is only the analysis by a Petri Net, it is possible only using course1.Sdata in Fig.3, but by this simulator, since visualization is a big feature, it has the basic menu of the Petri Net simulator shown in Fig. 4. The figure arrangement window of the screen right is a screen for arranging Place of Fig.3, and transition by hand. About an arc, course1.Sdata in Fig.3 is referred to and drawn automatically. The information on the position of Place and transition etc. is automatically generated as “course1.Ndata” in Fig.5 after all the Place and ends of transition arrangement. The portion shown in the gray of Fig. 5 shows the basic format of Ndata. The coordinates (x, y) of PLACE arranged using Sdata and TRAN are recorded

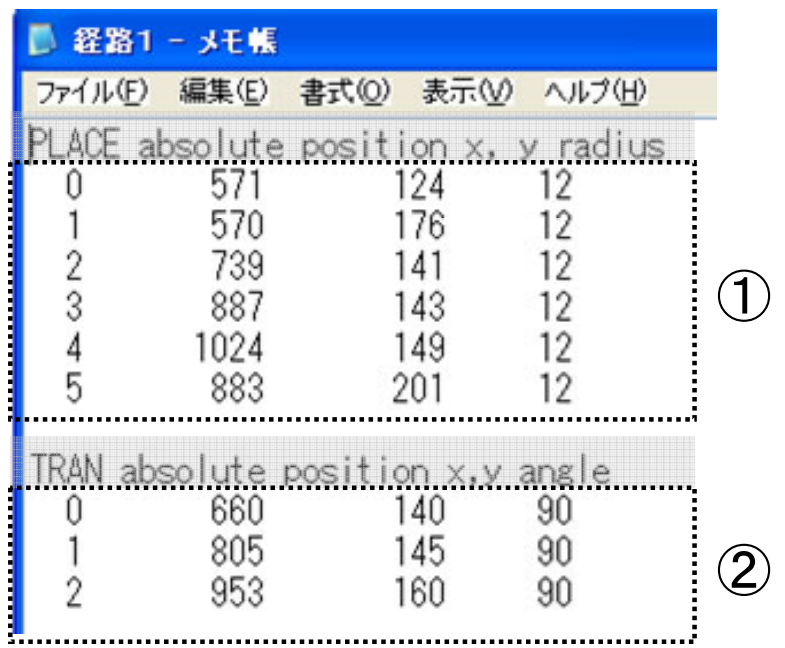

Fig. 5 Petri Net Ndata File

on the figure. And about PLACE, the radius of Place who drew is recorded on radius. The angle which an arc and transition cross is describing TRAN. Dashed line part (1) of Fig.5 expresses Place's radius as the main (x, y) coordinates which make the upper left of each place the starting point. Dashed line part (2) expresses angle of the coordinates ( $x$, y) of the central part of each transition, and transition and an arc. "Sdata" used as one-pair correspondence and "Ndata" are needed for execution of a simulator. "Insertion (I) of a background image" of the portion of (2) and a display (V) pull down menu is a menu for reading background images, such as a map, among the menu in

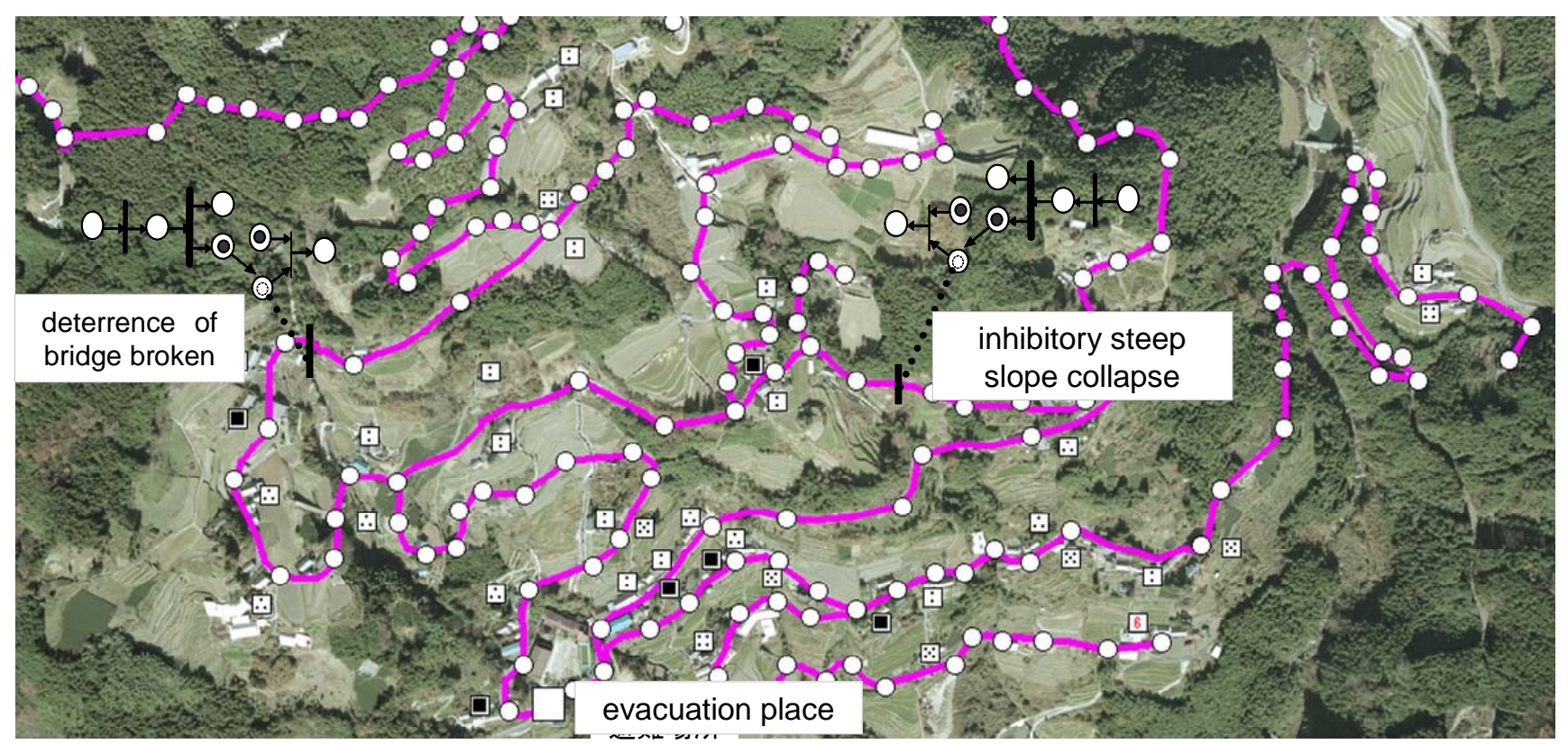

Fig.6 The Research Area of Kumakougen Town in Ehime Prefecture by Petri Net. 
Fig.4. It is the menus about a display and un-displaying of the arc of the Petri Net figure of the figure right, transition, etc. "a display and un-displaying" (D) of (3). These functions are used by the following clause.

\section{(3) Composition of the Petri Net simulation of the} evacuation plan scenario in the mountainous area

\section{a) The outline and basic net figure of the research region}

The research area is Kumakougen towni in Ehime Pref. This area is located in the point which branched on the branch from the national highway No. 33 line which is a trunk road, and the place-between-mountains slope is dotted with the residence. This local whole region is specified as the landslide dangerous area, and it is dotted also with the landslide disaster dangerous mountain stream and the landslide dangerous place. We are developing the evacuation plan scenario Petri Net simulation of the area mentioned above using the Petri Net simulator as previously mentioned (2). The Petri Net figure of the created application area is shown in Fig.6. The composition procedure of this network is described below. First, on space, in handwriting, like description of the refuge scenario by the Petri Net of Fig.1-2, next it stations transition and Place for the residence of the region, and a course, Sdata of the region is created according to the Sdata formal description format of the Petri Net of Fig.3. And Petri.exe is performed and created Sdata is read.

And Place and transition are arranged using the "arrangement of network" function of the basic menu of the

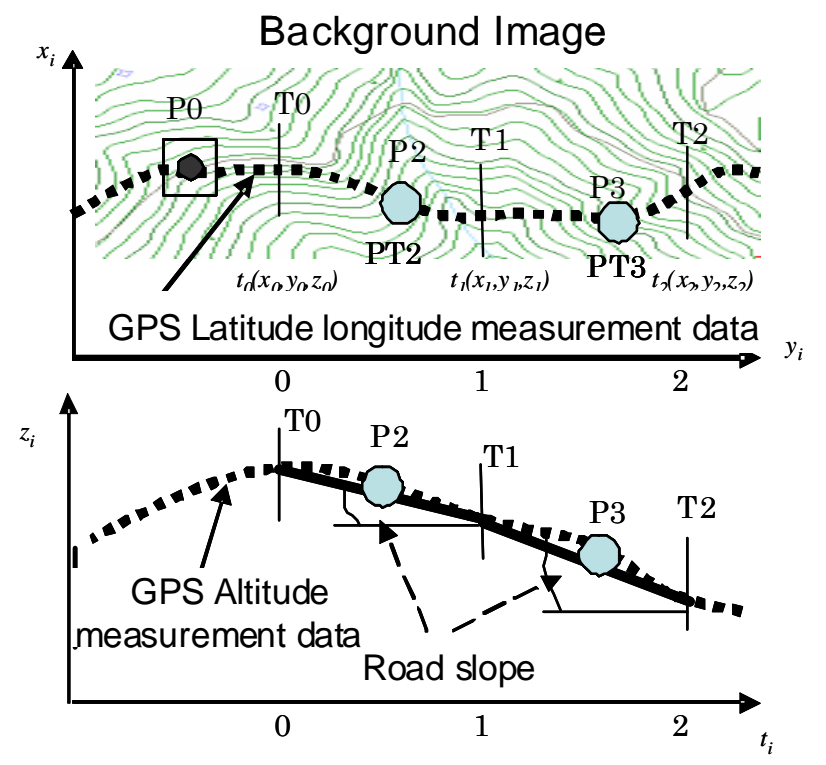

Fig.7 Concept of Place Timer Set Reflecting Travel Time of Slope by GPS Measurement Data
Petri Net simulator of Fig.4. Next, the aerial photograph ${ }^{11)}$ of the area concerned is displayed using an "insertion of background image" function. Place and transition are made to form into space correspondence on a background image finally. Completion of the above operation will save the position coordinate of Place and transition as Ndata in the format explained automatically in Fig.5. The data for simulators (Sdata, Ndata) was created in the above procedure. A prevention factor ${ }^{12)}$ is searched from a background image to this basic data, and the addition of disaster prevention, and a prior / posteriori measure against Fig.2 is added to Sdata. In Fig.6, only a house and route Place are displayed using a display and the function in which it does not display of a simulator. In the figure, square Place expresses housing Place, as Fig.1-2 described. Moreover, the number of tokens struck by those Place expresses the number of residents of the household. The big Place of the center bottom express the main gate of the evacuation area (elementary school which was closed down). The prior measure network is added to left-hand side and right-hand side with the addition of the prevention factor by bridge destruction damage and steep slope collapse damage, respectively. About this subnet, the network is specified using a display and the function in which it does not display of a simulator. As mentioned above, building the subnet of prevention and an evacuation route in a basic network is possible. Therefore, it suggests possibility of building the opinion and proposal in a planning by an addition and extension of a network

b) Reflection to the travel time (place timer) of a slope

We are performing development and application in urban areas of the evacuation plan scenario Petri Net simulation. Since the movement speed of a there is a flat ground, the value which broke the distance between transition by average movement speed is used for it as place timer. On the other hand, in the case of intermediate and mountainous, the geographical feature and the slope of a road affect a car and on foot movement speed. Then, the proposal about a setup of place timer in consideration of the calculation of migration length using portable GPS and a road slope is performed. The key map of the place timer grant which reflects the road slope is shown in Fig.7. Three-dimensional move coordinates which the dashed line in a figure measured by portable GPS: (latitude, longitude, altitude) are shown. T01 which means the distance between T0 and T1 can be calculated by the distance between the moving points concerned, and should just add and unite them in approximation. 
The value which broke the distance between $\mathrm{T} 0$ and $\mathrm{T} 1$ by movement speed is used for PT2 as place timer. However, since movement speed is influenced by a slope, it calculates a slope $\theta_{01}$ as the advanced difference between T0 and T1 of Fig.7 from latitude longitude. Average movement speed:v is calculated using authors' empirical equation (1) and (2) using the calculated slope. Therefore, what is necessary is just to give T0, distance:T01 (ascent) between $\mathrm{T} 1$, or the time broken by the value of (descent) to

$$
\begin{aligned}
& V_{a s}=-1.1498 \sin ^{2} \theta-0.1814 \sin \theta+1.4028 \mathrm{~m} / \mathrm{s}(1) \\
& V_{d s}=\text { const }(1.4) \mathrm{m} / \mathrm{s} \\
& \text { provided, }
\end{aligned}
$$

$V_{a s}:$ ascend time ( $\mathrm{sec}$ )

$V_{d s}:$ descend time ( $\mathrm{sec}$ )

$\theta$ : road slope

PT2 as place timer.

However, if residents' refuge action GPS data is collected, setting up actual travel time as place timer will also be considered.

\section{Construction of Moving Part Petri Net by GPS Measurement Data}

As stated for the purpose of research of Chapter 1, in order to collateralize the execution of disaster prevention planning, it is necessary to devise participation of the residents to a plan. We aims at grant of direct inclusion for the scenario simulation of residents' refuge act in this paper. In order to create the conventional plan scenario, Sdata which described Place and transition was created and Ndata was created by carrying out space arrangement of them to up to a background image. That is, Sdata and Ndata are in the relation corresponding to one pair. In this paper, as proposed by 2. (3), the transition coordinates of a land survey system, presumption of place timer by an empirical equation, and the direct thing for which place timer acquisition can be carried out were proposed from the three-dimensional position coordinate measured by portable GPS. That is to say, Sdata of a Petri system and Ndata are automatically generable from the measured data.

This can expect the practical use and deployment as a tool of the further public involvement, such as formation of instant reflection of residents' refuge act, and an addition of a new course. This clause describes the technical development for the formation of an automatic input to the

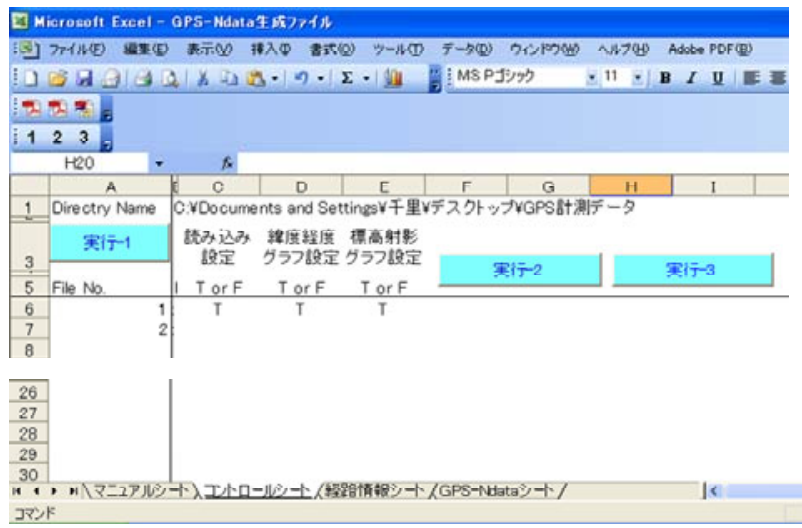

Fig.8 Control Sheet and Execution Button

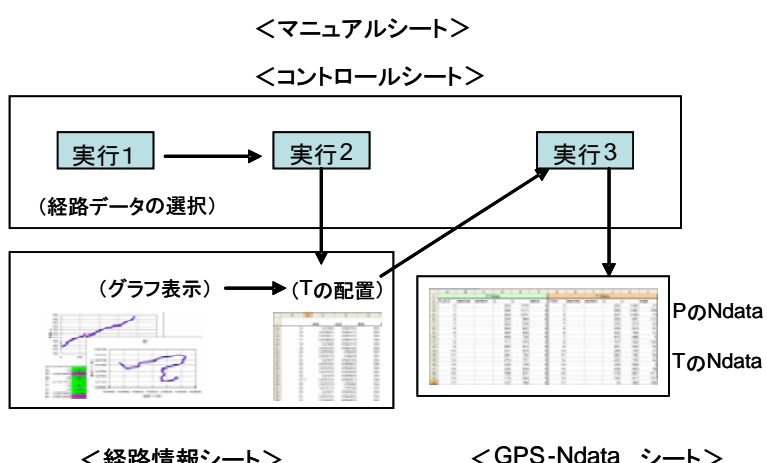

Fig.9 System Configuration of GPS-Ndata Generation File

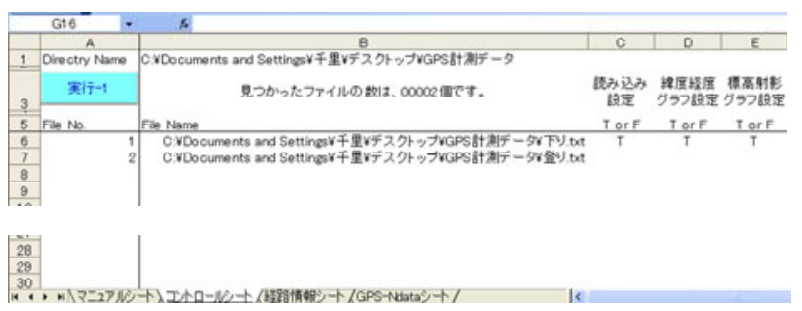

Fig.10 Specification of Display Graph of GPS Measurement Data File by Control Sheet

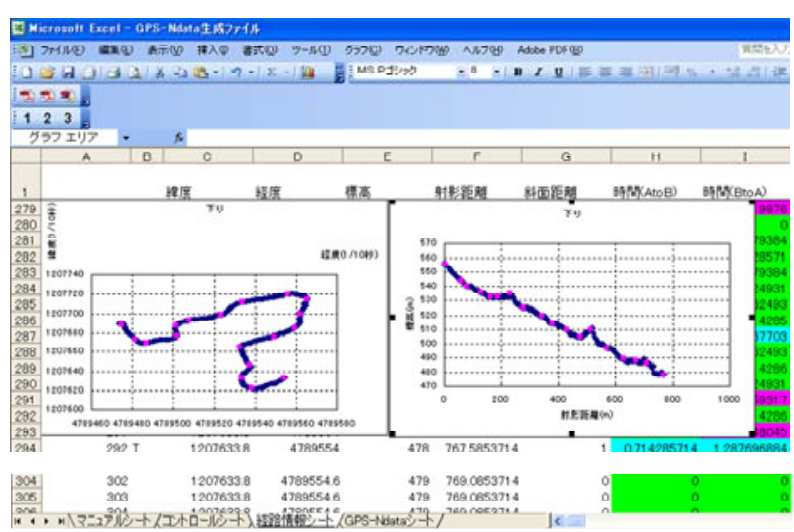

Fig.11 Arrangement of Transition by Channel 
Petri Net simulation of GPS data.

\section{(1) The generation system of Ndata of the moving} part network by GPS coordinate data

We walked along some routes of the research region shown in Fig.6 using portable GPS (EMPEX Map21), and GPS measurement data was extracted. Measurement data is saved in CF memory and reads data into a personal computer using a card reader. Since the interval of preservation data was a minimum of 2 seconds, this model extracted the measurement data in every 2 seconds. And only latitude and longitude, and the required data of being advanced are extracted from measurement data on a personal computer, and it saves by text format. The control sheet and execution button of a GPS-Ndata generation file which were developed are shown in Fig.8. The execution button of the control sheet of Fig.8 and the relation of each sheet serve as a system configuration of Fig.9.

Below, the function of each sheet is explained.

\section{$<$ Manual sheet $>$}

While arranging transition to Ndata of a GPS coordinate system, i.e., the locus of GPS measurement data, and arranging the halfway point automatically as Place from the measurement data saved by text format. The procedure for acquiring the travel time between transition as place timer is specified.

\section{$<$ Control sheet $>$}

It is a sheet which controls the data between sheets, such as an input condition and an output condition for selection of course data, selection of a graphical representation, arrangement of Tran John, and generation of GPS-Ndata. As shown in Fig.9, it processes by clicking various execution buttons. In Fig.10, by the 1st line, a folder is specified and the GPS measurement data in a folder, descent.txt, and ascent.txt are displayed.

The existence of drawing of selection of a file, latitude longitude graph, and advanced graph is specified by $\mathrm{T}$ and F.

\section{$<$ Channel information sheet $>$}

As shown in Fig.11, it has a transition arrangement function to a graphical representation and measurement data. As it was called two or more courses and the course according to attribute, GPS measurement data attaches a file name, respectively, and saves it at a folder. While first specifying a course preservation folder, displaying a file list

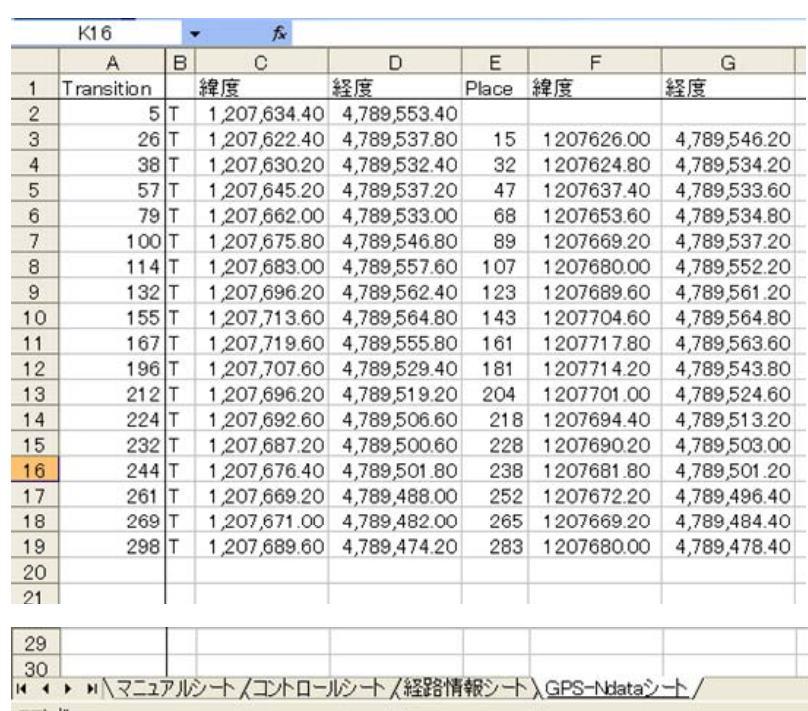

Fig. 12 T to GPS-Ndata Sheet, Coordinates Output of $\mathbf{P}$

and choosing required move part measurement data, the existence of a display of the latitude and the longitude graph of Fig.8, and advanced graph is inputted. If the execution 2 of a control sheet is clicked, while the latitude, the longitude, and the advanced information on the data chosen as the channel information sheet will be displayed by tabular form, the specified graph is also displayed on this sheet. Transition is specified looking at form and slope graph next, as given an outline in Fig.7. If the execution 3 of a control panel is clicked after specifying all the transition, as shown in Fig.12, the transition Place coordinates of a GPS coordinate system will be outputted to a GPS-Ndata sheet. A series of above operations show that the transition and Place's land survey system Ndata which are the constituent factors of GPS measurement data to Petri Net simulation data can be created.

\section{(2) Move part Net generation on the construction of Sdata, and a background image}

The preceding clause described the procedure which creates the land survey system Ndata by the transition arrangement reflecting a road slope from portable GPS measurement data. This clause describes the construction of Sdata from conversion to Ndata of a Petri system using GPS-Ndata generated by (1). Fig.13 expresses the Petri system coordinate conversion sheet of a Sdata generation file. The system configuration of this file consists of a Petri system coordinate conversion sheet, a Ndata generation sheet, and a Sdata generation sheet like GPS-Ndata generation file using the macro function of Excel, as it is in the sheet name of Fig.13. Below, the 
function of each sheet is explained.

\section{<Petri system coordinate conversion sheet>}

As shown in Fig.13, (1) whose input units are (1) and (2) inputs the coordinates $(\mathrm{x}, \mathrm{y})$ of the datum points $\mathrm{A}$ and $\mathrm{B}$ where a simulator screen corresponds as the latitude longitude of the datum points $\mathrm{A}$ and $\mathrm{B}$ of the background image used by a simulator. The portion of (2) is a transition coordinates output to the GPS-Ndata sheet of Fig.12.This portion is inputted automatically. The coordinates of GPS-Ndata are changed and outputted to a Petri system pixel coordinate at(3).By this operation, the transition coordinates of a land survey system and the Place coordinates are changed into the Petri system coordinates on a background image.

\section{$<$ Ndata generation sheet $>$}

Fig.14 shows the sheet which generates the Petri system Ndata (position of Place and the transition by the format of Fig.5) using the land survey system data of the position coordinate of Petri system transition Place of (3) of Fig.13, and Place of Fig.12. The shading portion of Fig.13 is a format portion of Ndata. 18 after declaration of PLACE is the number of Place. Similarly, 17 after declaration of TRAN is the number of transition. As for one side and the number of Place into which the number of transition has inputted the number of lines of transition as an argument, +1 transition serves as the Place number from the relation between Place and transition. However, since the middle is set up as Place after deciding the position of transition as shown in Fig. 7, it has been set as Place of the start of transition, and the end, the p0 and p18 in Fig.14 (Place of the beginning and the last) so that the position coordinate of transition may enter. The radius of Place of Ndata: radius is considering 12 as the default. Here, about angle:angle of transition and an arc, it has given in the formula so that it may intersect perpendicularly with the arc from Place.

\section{$<$ Sdata generation sheet $>$}

Fig.15 shows the Sdata generation sheet which made the argument the number of Place of a Ndata generation sheet, and transition. A gray portion is a Sdata format. The value of place timer is inputted into (1). (2) and (3) are the input-and-output relations of Place to transition. Since the number of transition corresponds continuously with Place, the course data of a move part can also be automating the input of (2) and (3). At the end, the result of having performed simulator Petri.exe is shown in Fig.16 using Sdata and Ndata which were generated. As mentioned above, when carrying out GPS measurement of residents' refuge act, it was able to propose that generation of Sdata and Ndata which are input data of a Petri Net could be easily performed by the system to propose. This can expect the practical use and deployment to the formation of direct participation to residents' plan which we

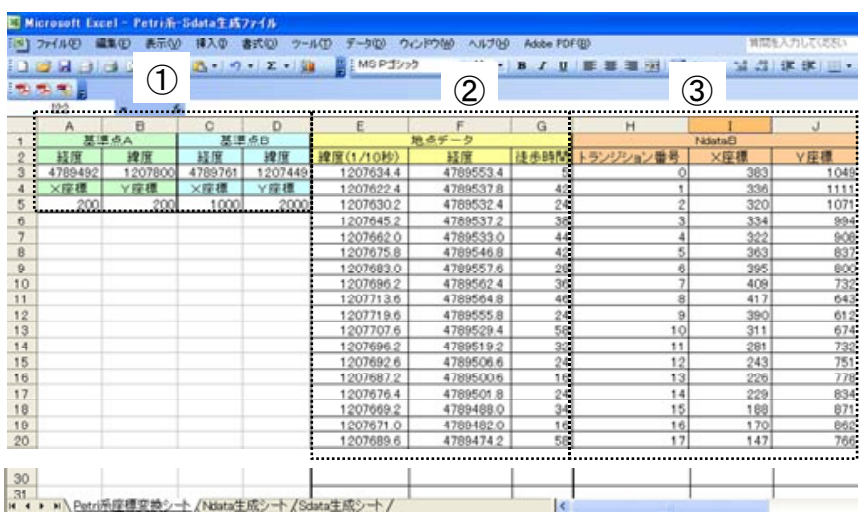

Fig. 13 Petri System Coordinate Conversion Sheet

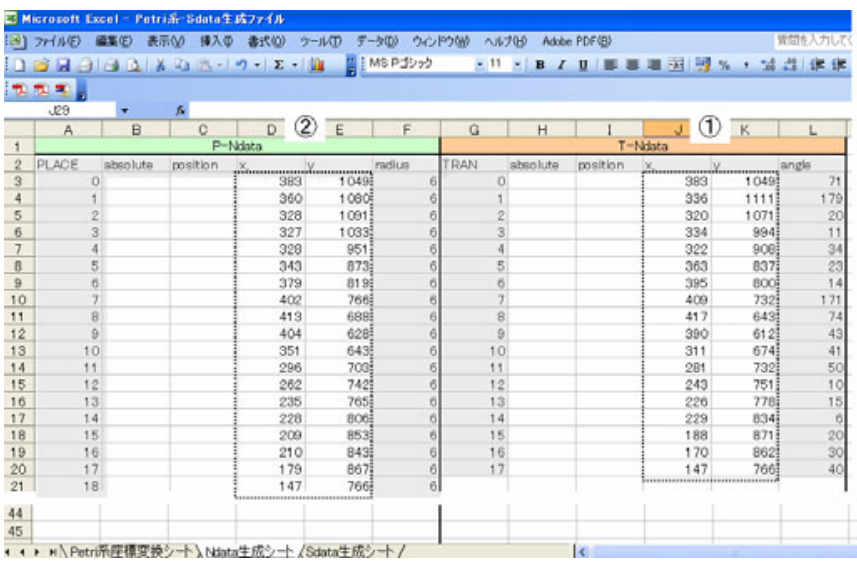

Fig. 14 Ndata Generation Sheet

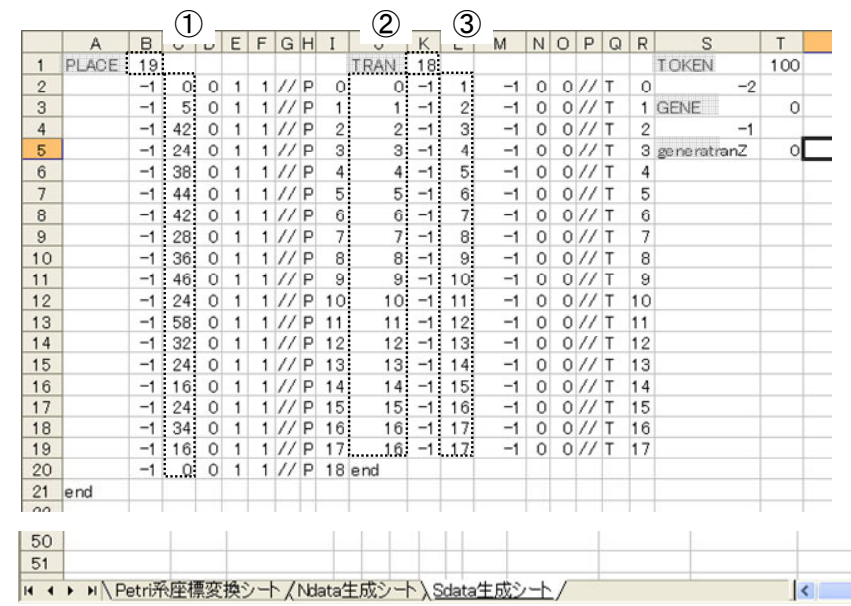

Fig. 15 Sdata Generation Sheet 
aim at. In Chapter 4, authors perform comparison and verification with the scenario proposed in Fig.6, and the evacuation plan scenario by GPS data.

4. Direct Participation Support of Residents to Evacuation Plan Scenario in intermediate and mountainous area

We have proposed the evacuation plan scenario by the Petri Net shown in Fig.6. Since this plan is a proposal by a specialist, it compares the plan scenario proposed as residents' refuge act, and clarifies both relations. First, the form of the course Petri Net network by residents' refuge action data is compared with the refuge scenario which we planned. Next, the place timer value calculated by the speed type which authors assume is compared with the place timer value given by actual travel time. Finally the on foot course which residents point out is added, and the subject towards expansion of future public involvement is argued.

(1) Route verification of the evacuation plan scenario by portable GPS data

It is a level of the beginning of public involvement to reflection of opinion and criticism of residents to an evacuation plan scenario. For example, about new indication of a prevention part, or reflection of the opinion of release of prevention, a subnet can include in Sdata and it can respond instancy by operation of release of prevention network. By this clause, the network created from the aerial photograph is first compared with the subnet measured by portable GPS. Fig.17 shows the track data of A to B on foot, an examinee equipped with portable GPS in his 40's. Fig.17 stations only Place using a display and the function in which it does not display of the menu panel of a simulator. Fig.17 shows that Place is stationed on the course of the proposed pink color. It is proved that the proposed plan scenario is reflecting residents' refuge act. That is to say, it also means that all the networks can be created by walking to residents and getting them to take refuge from all the courses. On the way of a refuge act, Residents will discover a prevention factor and will become possible to incorporate a prevention network by themselves. The place timer value using the speed type which makes a parameter next the slope which authors propose was compared with the place timer value by a subject's real land survey. A result is shown in Table 1. As the result of calculation of the correlation coefficient from Table 1, the good result of $\mathrm{R}=0.976$ was obtained. However, a subject

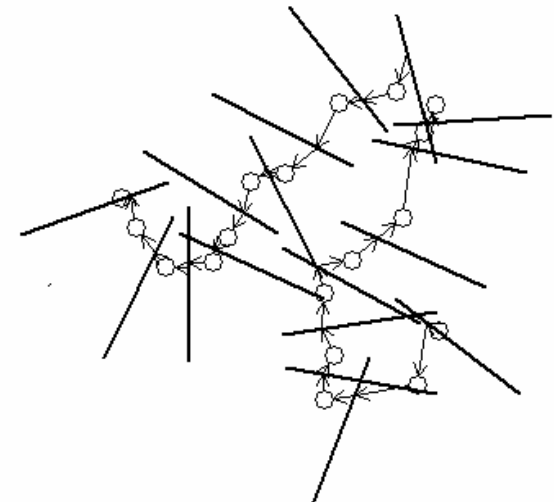

Fig. 16 Generation of Subnet by Sdata Generation File

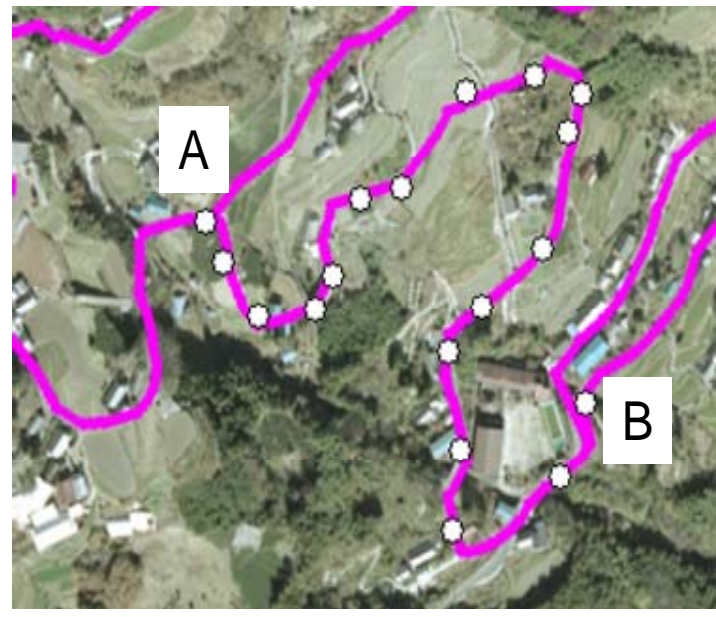

Fig.17 Comparison of Plan Scenario and GPS Measurement Subnet

Table 1 Comparison of Model and Actual Measurement

\begin{tabular}{|l|r|r|}
\hline $\begin{array}{l}\text { place } \\
\text { timer }\end{array}$ & $\begin{array}{l}\text { time on foot } \\
\text { (descend):second }\end{array}$ & $\begin{array}{l}\text { model formulation } \\
\text { (descend) :second }\end{array}$ \\
\hline PT1 & 5 & 10 \\
\hline PT2 & 42 & 49 \\
\hline PT3 & 24 & 24 \\
\hline PT4 & 38 & 39 \\
\hline PT5 & 44 & 42 \\
\hline PT6 & 42 & 45 \\
\hline PT7 & 28 & 32 \\
\hline PT8 & 36 & 34 \\
\hline PT9 & 46 & 43 \\
\hline PT10 & 24 & 27 \\
\hline PT11 & 58 & 61 \\
\hline PT12 & 32 & 35 \\
\hline PT13 & 24 & 26 \\
\hline PT14 & 16 & 16 \\
\hline PT15 & 24 & 26 \\
\hline PT16 & 34 & 36 \\
\hline PT17 & 16 & 15 \\
\hline PT18 & 591 & 55 \\
\hline 合計 & & 617 \\
\hline
\end{tabular}


is the limited data of one person, and I think that an old person etc. also needs examination of the model type in consideration of a subject attribute.

\section{(2) Recombination support of the evacuation plan} scenario by residents' course proposal

This clause describes the support for including the proposal by residents' experience knowledge in an evacuation plan scenario. The evacuation plan scenario of Fig.6 is a proposal by the specialist on authors. We sent this refuge scenario to residents, and had the evacuation route illustrated at the time of disaster. As a result, it became clear that there are six on foot limited evacuation routes. In order to rearrange the escape route for evacuees of these resident proposals to the refuge scenario of Fig.6, portable GPS was equipped and six courses were moved on foot. Using the construction of the move part Petri Net according those data to 3.GPS measurement data, as shown in Fig.17, Sdata corresponding to a background image and Ndata were generated. And if each data is added to Sdata of Fig.6, and Ndata, the scenario of the course which residents propose can be added. The additional example of the data to Sdata of Fig.6 and Ndata is shown in Figs.18 and 19. Finally, the reflection figure to the plan scenario of six resident proposal courses is shown in Fig.20. As mentioned above, by our having proposed and using a system was able to show that an opinion and criticism of residents' refuge action, an addition of a course, etc. could be easily reflected in a plan scenario.

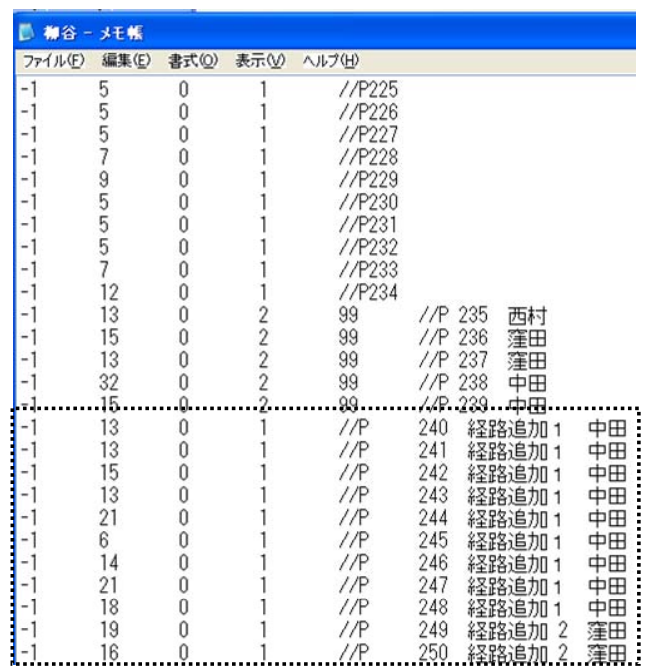

Fig. 18 Description of Additional Route to Sdata

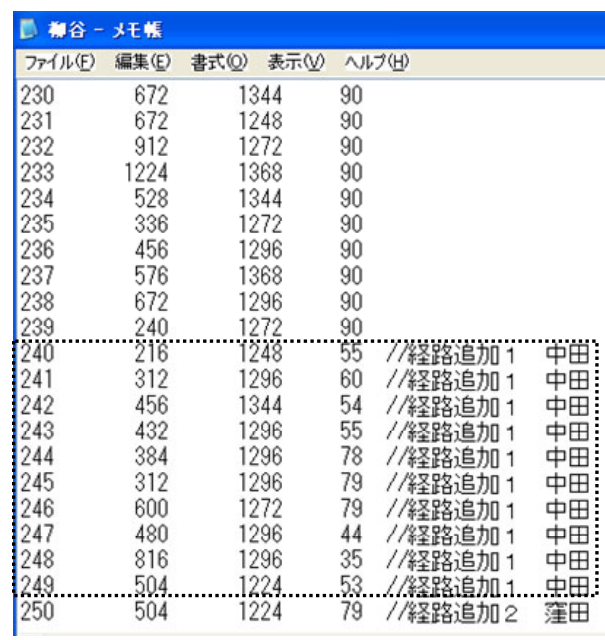

Fig. 19 Description of Additional Route to Ndata

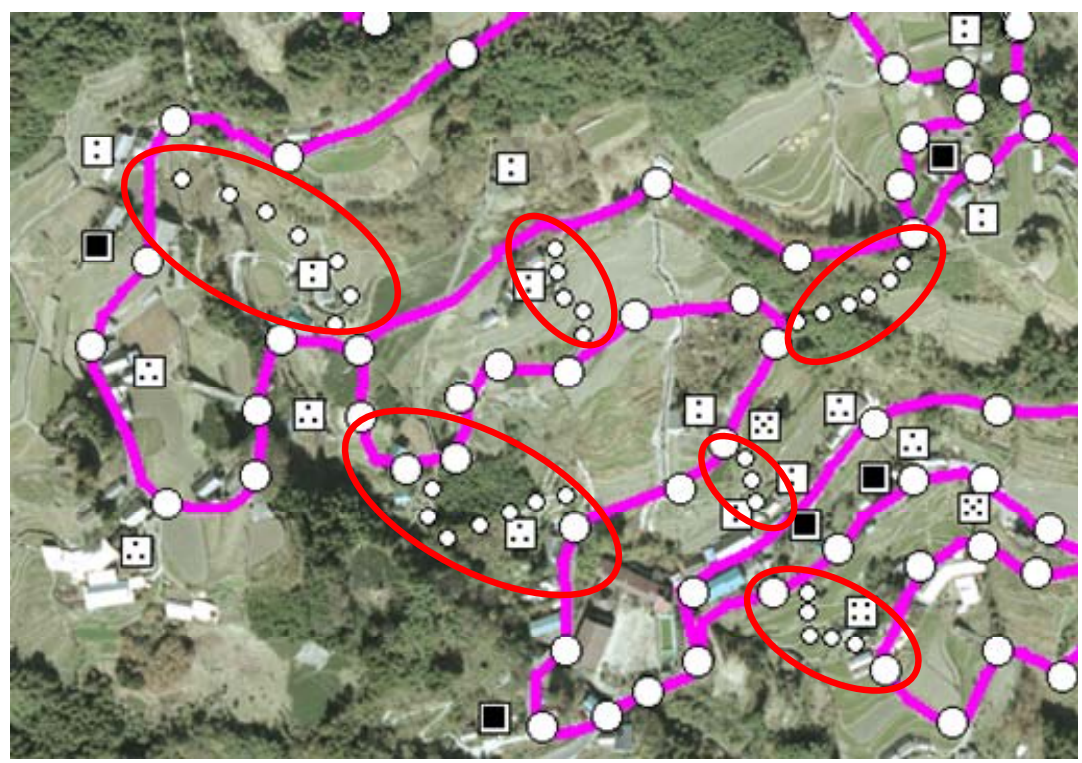

Fig. 20 Reflection to a Scenario Plan of Residents' Proposal Route 


\section{Conclusion}

The main body of an evacuation plan is resident and we claim that the tool which supports the independent measure is important. Therefore, the evacuation plan scenario simulator in which construction and execution are possible on a background image has been developed based on the Petri Net which is rich in descriptiveness and extendibility. Namely, by using a digital map, an aerial photograph, a satellite imagery, etc. as a background image in a city area With the refuge prevention situation by building collapse or fire spreading disaster, and intermediate and mountainous area. The developed system is enabling remembrance of the refuge prevention situation by bridge breakage or slope collapse. And the supportive tool which enables eye-minded simulation execution on the background image in which experience of resident works by performing an evacuation plan scenario and its Petri Net composition has been proposed based on those remembrance. The feature of this tool is in simple and intelligibility of the basic principle of description and driving of an object system which a Petri Net possesses.

Furthermore, we think that fixed contribution is carried out to residents' independent measure support in that an understanding and an utterance are conjointly made comparatively easily also at residents with the visibility which an above-mentioned experience commits. However, since the independency is only a restrictive thing that bottoms out at the level of the reflection of an opinion, its technical deployment to much more participation support is desirable. This research reported improvement of the participation support level of this system under cooperation with use of portable GPS information. First, in 2., the basic principle of the Petri Net simulator which we have developed, and the application example to the evacuation plan scenario simulation in intermediate and mountainous area were outlined. Moreover, practical use of three-dimensional GPS data was described here as reflection technology to place timer of vertical interval information peculiar to intermediate and mountainous area.

In Chapter 3, the system proposal which generates the basic partial Petri Net of an evacuation plan scenario from this GPS data was performed conversely. That is, use of portable GPS showed that the reflection of residents' refuge action for a direct scenario simulation was attained.

And in Chapter 4, the case study was shown and improvement of the more direct participation to residents' evacuation plan was discussed. A future subject is having stopped at analysis of the restrictive course by a one subject this time. We would like to collect the course data by the more residents, and we would like to verify the accuracy of the empirical equation by using our slope parameters. Temporarily, it will lead to recognition of the credibility of the proposed plan scenario, if there is no big deviation in an empirical equation and resident data. On the contrary, if reproducibility is scarce, I think that the meaning using the system proposed in that it is necessary to set up place timer by a resident individual's refuge speed is significant. Anyway, another subject may be in sight by having developed and utilizing a system under citizens' participation in municipal affairs.

Lastly, we would like to perform extraction of the subject by actual practice, and approach for problem solving in the meaning.

\section{Reference}

1) Central Disaster Prevention Council: the basic policy about promotion of the national movement which reduces disaster damage, 2006.4.21.

2) Central Disaster Prevention Council: the synthesis emergency drill fundamental principles in the Heisei 18 fiscal year, 2006.4

3) Kiminori Meguro, Yasunori Haga, Fumio Yamazaki, Tsuneo Katayama: Application to the refuge action simulator of a virtual reality, Journal of Japan Society of Civil Engineers, No.556/I-38, pp.197-207, 1997.4.

4) Fumio Yamazaki: "Real time Control Technique of an Infrastructure System, chapter 9, Construction of the refuge action space model at the time of disaster", scientific inquiry expense specification domain result of research report, 2000.3

5) Yasuo Chikada, Satoshi Hirose, Takayoshi Kido : Construction of the Walk Simulation Model using CA, Proc. of Applied Computing in Civil Engineering, Vol.9, pp.19-30, 2000.10.

6) Hideyuki Nakanishi, Satoshi Koizumi, Hiroshi Ishiguro, Tohru Ishida: Toward the refuge simulation by citizen participation, Journal of the Japanese Society for Artificial Intelligence No.Vol.18-6 a, pp.643-648, 2003.

7) Noboru Kimata、 Ikuo Sumi : Fundamental Research on the Petri Net Simulation of the Prevention Factor of a Fire-fighting Disaster Prevention System, Proc. of Infrastructure Planning, No.14, pp.393, 1997.

8) Kozo Hori, Noboru Kimata : Development of Petri Net Simulation for Emergency Vehicle in Case of the Earthquake on a Background Image, Infrastructure Review,Vol.30, CD-ROM, and November, 2004

9) Tohru Futagami, Noboru Kimata: Development of the Scenario Simulation for the Emergency and Evacuation Plan Support in an Intermediate and Mountainous Area, Infrastructure Review, 
Vol.30, CD-ROM, November, 2004.

10)Tohru Futagami, Noboru Kimata: Development of Information System for the Emergency and Evacuation Plan Support in Intermediate and Mountainous Area, Proc. of Applied Computing in Civil Engineering, Vol.29, pp.29-32, 2004.

11) Kumakougen town's civil engineer division in Ehime Prefecture: Aerial Photograph in Yanadani.

12) Ehime prefecture's civil engineering division :

Sand Disaster Risk Map,

http://www.pref.ehime.jp/doboku/doboku2/kabetu/sabo/dm 